\title{
THE DIRECT LATERAL APPROACH TO THE HIP
}

\author{
KEVIN HARDINGE \\ From the Centre for Hip Surgery, Wrightington Hospital, Lancashire
}

\begin{abstract}
A direct lateral approach to the hip is described which allows adequate access for orientation of the implant, for the insertion of the cement and for the correction of discrepancy in leg length. An anatomical observation was made that the gluteus medius muscle is inserted into the greater trochanter by a tendon and that the axis of the shaft of the femur lies anterior to the main bulk of the muscle which was left undisturbed.
\end{abstract}

In 1954 McFarland and Osborne described a new surgical approach to the hip which was based on their anatomical observation that the gluteus medius and vastus lateralis were in functional continuity through the thick tendinous periosteum covering the greater trochanter. The patient is placed on his side and the gluteus medius and vastus lateralis are detached from their posterior borders and the combined muscle swung forward like a bucket handle. This procedure normally involves detaching some spicules of bone or in some cases a thin shell of bone from the lateral aspect of the trochanter which is then taken forward.

In total hip replacement, the lateral approach with the patient in the supine position offers distinct advantages for orientation of the implant, insertion of cement and correction of the discrepancy in leg length. A new surgical approach to the hip is described which takes advantage of the fact that the insertion of the gluteus medius to the greater trochanter is by a strong tendon which is wide in its anterior half and follows a crescent shaped insertion (Fig. 1). At the apex of the greater trochanter the fibres of the gluteus medius lie horizontally. Furthermore the axis of the shaft of the femur is continuous with the anterior half of the greater trochanter which is revealed when the tendon of the anterior half of the gluteus medius is divided.

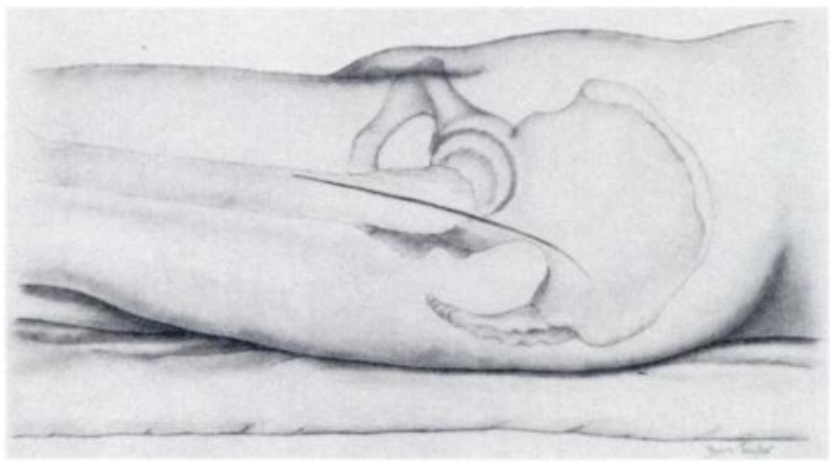

Fig. 2

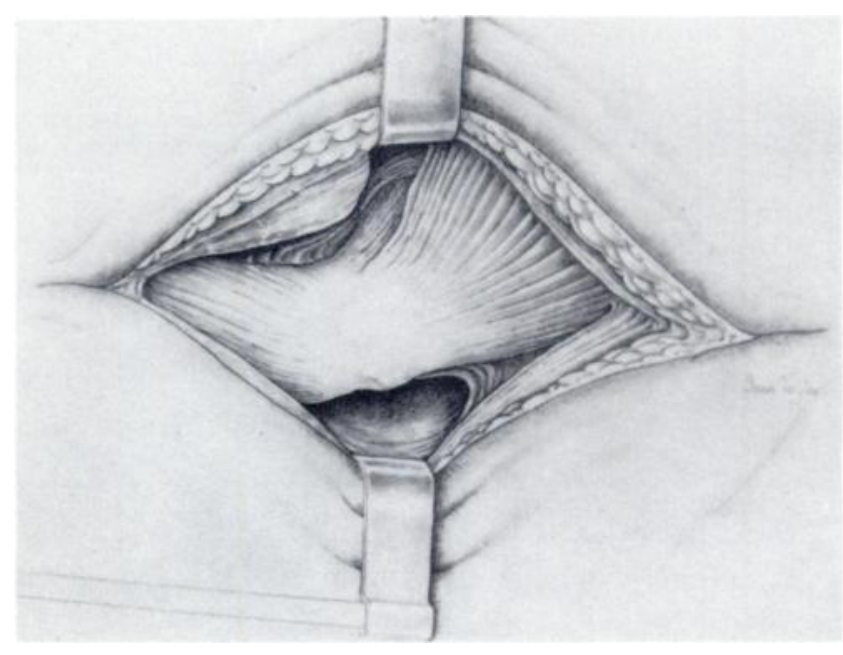

Fig. 1

Attachment of the gluteus medius to the greater trochanter by a thick mobile tendon. This tendon curves round to the apex of the trochanter.

\section{METHOD}

The patient is placed in the supine position with the greater trochanter lying at the edge of the table, thus freeing the muscles of the buttock from the table. The skin incision has the greater trochanter as its midpoint longitudinally and extends eight centimetres parallel to the shaft of the femur along its anterior border. Proximally the incision extends in a posterior direction and ends at the level of the anterosuperior iliac spine, although in patients with much muscle the

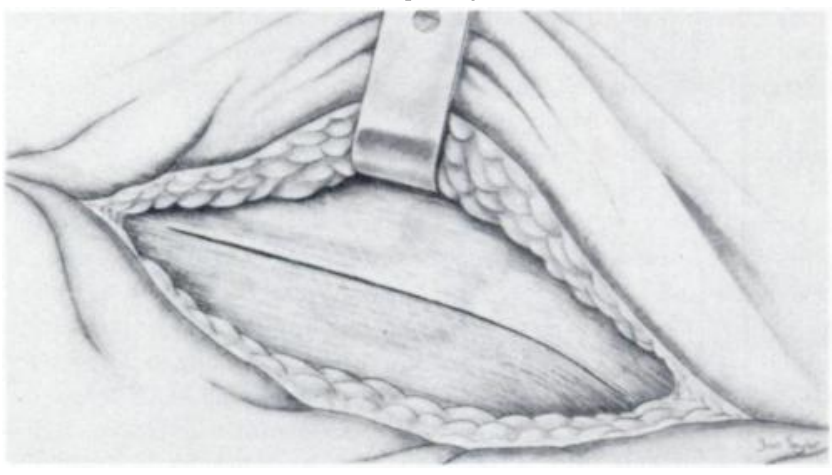

Fig. 3

Figure 2-Skin incision (a lazy J) parallel to the femur distally, curving posteriorly with the greater trochanter at its midpoint. Figure 3-Incision of the fascia latae in line with the skin incision.

Kevin Hardinge, MChOrth, FRCS, Consultant Orthopaedic Surgeon, Centre for Hip Surgery, Wrightington Hospital, Appley Bridge, Near Wigan, Lancashire. WN6 9EP, England.

(1) 1982 British Editorial Society of Bone and Joint Surgery 0301-620X/82/1018-0017 $\$ 2.00$ 


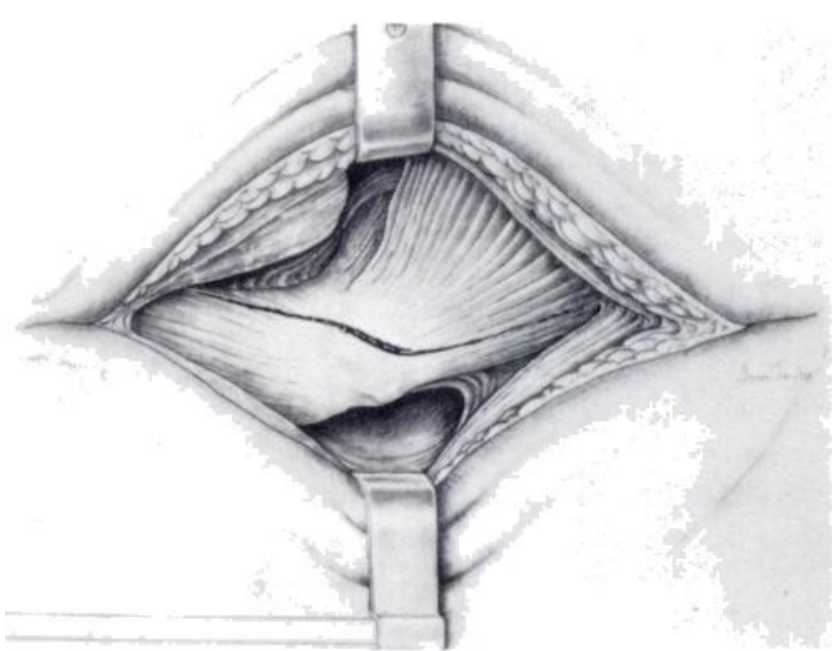

Fig. 4

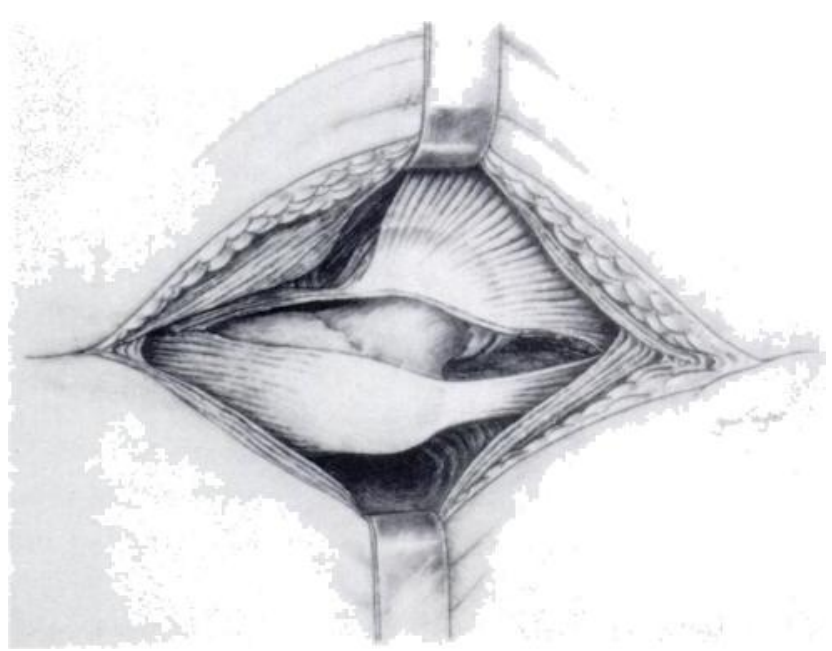

Fig. 5

Figure 4-Incision of the tendon of the gluteus medius leaves a cuff of tendon attached to the greater trochanter to facilitate closure and early function. Figure 5-Adduction of the thigh causes the anterior half of the gluteus medius to swing forward to reveal the gluteus minimus.

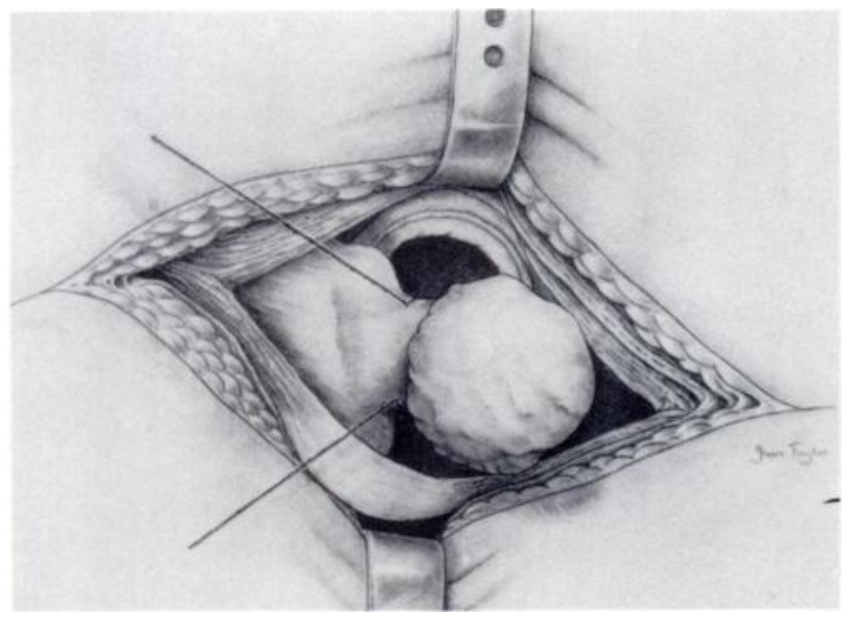

Fig. 6

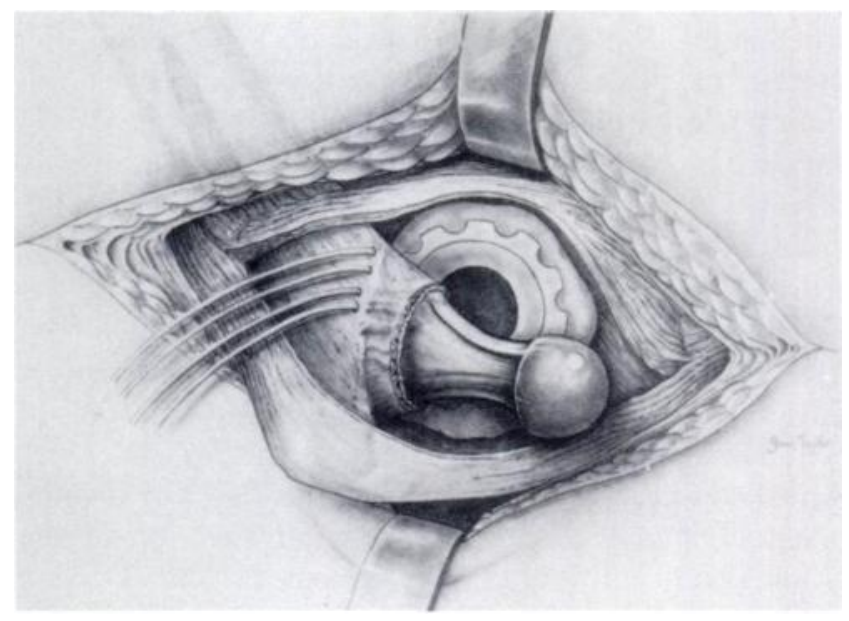

Fig. 7

Figure 6-The neck of the femur can be divided with a Gigli saw since the axis of the neck and shaft of the femur are anterior to the apex of the trochanter and the main mass of the gluteus medius which is left undisturbed. Figure 7-Closure of the joint after arthroplasty. Firm sutures are passed through the confluent ligament of Bigelow and the tendon of the gluteus minimus.

incision should be extended a further four centimetres proximally (Fig. 2).

The gluteal fascia and iliotibial band are exposed and divided along the midlateral line along the whole length and in the same direction as the skin incision (Fig. 3). The tensor fascia latae is retracted anteriorly and the gluteus maximus posteriorly. Any fibres of the gluteus medius which arise from the deep surface of the gluteal fascia are pushed off by blunt dissection.

The greater trochanter thus presents in the centre of the incision; occasionally a bursa lies over it and needs to be excised. The insertion of the gluteus medius follows a crescent shaped course on the greater trochanter and the mobility of the tendon can be verified by blunt dissection. A small prominence lies at the uppermost end of the ridge of the vastus lateralis and starting at this point the tendon of the gluteus medius is incised using a diathermy needle but leaving a cuff of tendon still attached to the greater trochanter. The incision curves around the greater trochanter until it reaches the apex where it is extended proximally along and between the fibres of the gluteus medius, not cutting across them (Fig. 4). Distally the incision passes down to the bone through the vastus lateralis near the anterior surface of the femur. Bleeding in the vastus lateralis from the transverse branch of the lateral circumflex artery is controlled by diathermy.

The thigh is then brought across the body and this causes the

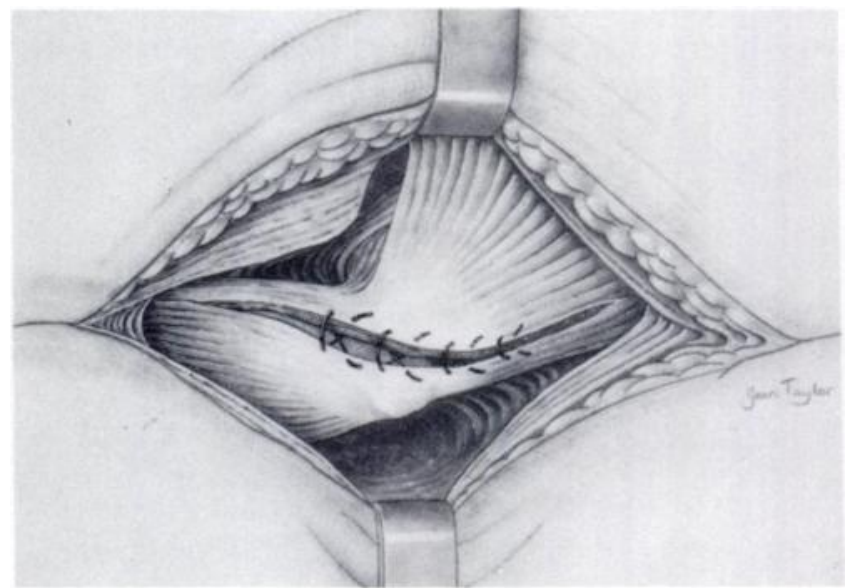

Fig. 8

Closure of the gluteus medius tendon allows early mobilisation with partial weight-bearing. 
anterior half of the gluteus medius to swing forwards (Fig. 5). Still using the diathermy needle, a small portion of the vastus lateralis arising from the intertrochanteric line is separated, and the tendinous insertion of the anterior portion of the gluteus minimus and the ligament of Bigelow separated from their ridge on the anterior aspect of the neck of the femur. With further adduction of the thigh, the capsule of the hip comes into view and is divided along the circumference with a further radial cut into the limbus of the capsule at the posterolateral aspect of the head of the femur. Dislocation of the head is achieved by full adduction and external rotation of the thigh. A good view of the acetabulum and shaft of the femur is obtained with routine retraction; capsular excision is not required. It should be noted that the posterior portion of gluteus medius with its thick tendon is left undisturbed (Fig. 6)

After the work in the joint has been completed the closure is made in layers. In the first layer the iliofemoral ligament is reattached using strong braided nylon suture which passes through the long ridge of the anterior aspect of the neck of the femur using a crotchet type of awl (Fig. 7). The second layer consists of the tendinous part of the gluteus medius which is sutured with braided nylon. These sutures bring tendon to tendon producing a strong seam which allows early mobilisation (Fig. 8) at two days, although as in all total hip replacements, partial weight-bearing is advised for up to nine weeks using elbow crutches to protect the bond between the cement and bone. The third layer consists of the iliotibial band and the gluteal fascia which are sutured using similar braided nylon.

\section{DISCUSSION}

The direct lateral approach to the hip with the patient in the supine position offers the optimal conditions for orientation of the implant, cementation and correction of any discrepancy in leg length. In the trochanteric osteotomy performed by Brackett (1912), the gluteus medius was retracted intact to give the widest exposure of the joint; this is particularly valuable in complex anatomical derangements and revision operations. The osteosynthesis of the greater trochanter remains a difficult surgical task and there can be troublesome symptoms after operation due to breakage of the wire, trochanteric detachment and bursitis.

The McFarland-Osborne approach depends upon detaching the posterior aspects of the vastus lateralis and gluteus medius. Small amounts of bone usually need to be chiselled up from the lateral aspect of the trochanter to preserve and in some cases to create the thick tendinous periosteum of the combined muscle layer. This procedure necessitates the patient being on his side but this position leads to difficulties in the orientation of the implant and in the correction of the discrepancy in leg length.

In this new direct approach the posterior part of the gluteus medius muscle with its thick tendon is left intact. The suture of the muscle is tendinous and resembles the medial parapatellar incision of the knee, where the medial capsule can be regarded as the tendon of the vastus medialis. Division and suture of this tendon is widely practised in all knee operations. However, since the posterior part of the gluteus medius muscle is not disturbed there is a limitation on the amount of distraction that is possible with this approach. Where a wide exposure is needed as in revision operations, wide distraction of the joint is only possible where the trochanter has been detached, otherwise unacceptable stretching of the gluteus medius will result.

I would like to acknowledge the help and encouragement and lively discussions with Mr Geoffrey Vaughan Osborne, former teacher, colleague and friend over many years for stimulating me to find an approach to the hip. I would also like to thank Ms Joan Taylor in the Department of Medical Illustration, the Royal Preston Hospital, Fulwood, Preston.

\section{REFERENCES}

Brackett EG. A study of the different approaches to the hip-joint with special reference to the operations for curved trochanteric osteotomy and for arthrodesis. Boston Med Surg J 1912;166:235-242.

McFarland B, Osborne G. Approach to the hip: a suggested improvement on Kocher's method. J Bone Joint Surg[Br] 1954;36-B:364-7. 